\title{
Nuclear Data Target Accuracies for Generation-IV Systems Based on the Use of New Covariance Data
}

\section{International Conference on Nuclear Data for Science and Technology 2010}

G. Palmiotti

M. Salvatores

M. Assawaroongruengchot

M. Herman

P. Oblozinsky

C. Mattoon

\section{April 2010}

This is a preprint of a paper intended for publication in a journal or proceedings. Since changes may be made before publication, this preprint should not be cited or reproduced without permission of the author. This document was prepared as an account of work sponsored by an agency of the United States Government. Neither the United States Government nor any agency thereof, or any of their employees, makes any warranty, expressed or implied, or assumes any legal liability or responsibility for any third party's use, or the results of such use, of any information, apparatus, product or process disclosed in this report, or represents that its use by such third party would not infringe privately owned rights. The views expressed in this paper are not necessarily those of the United States Government or the sponsoring agency. 


\title{
NUCLEAR DATA TARGET ACCURACIES FOR GENERATION-IV SYSTEMS BASED ON THE USE OF NEW COVARIANCE DATA
}

\author{
G. Palmiotti**1, M. Salvatores ${ }^{12}$, M. Assawaroongruengchot ${ }^{1}$, M. Herman ${ }^{3}$, P. Oblozinsky ${ }^{3}$, C. Mattoon ${ }^{3}$ \\ 1 Idaho National Laboratory USA \\ 2 Commissariat à l'Energie Atomique, Cadarache France \\ 3 Brookhaven National Laboratory USA \\ ${ }^{*}$ Corresponding author. E-mail : Giuseppe.Palmiotti@inl.gov
}

A target accuracy assessment using new available covariance data, the AFCI 1.2 covariance data, has been carried out. At the same time, the more theoretical issue of taking into account correlation terms in target accuracy assessment studies has been deeply investigated. The impact of correlation terms is very significant in target accuracy assessment evaluation and can produce very stringent requirements on nuclear data. For this type of study a broader energy group structure should be used, in order to smooth out requirements and provide better feedback information to evaluators and cross section measurement experts. The main difference in results between using BOLNA or AFCI 1.2 covariance data are related to minor actinides, minor $\mathrm{Pu}$ isotopes, structural materials (in particular Fe56), and coolant isotopes (Na23) accuracy requirements.

KEYWORDS: Covariance, Target Accuracy, Sensitivity, Integral Parameters

\section{INTRODUCTION}

As new high fidelity covariance data become available they can be used to assess nuclear data target accuracies for future innovative nuclear systems. The new covariance data produced at BNL (with the support of LANL and ORNL) and called AFCI 1.2 [1], have been used to update the findings of the NEA/WPEC Subgroup 26 [2].

Several advanced nuclear systems have been considered and uncertainties for the main integral design parameters (criticality and reactivity coefficients, power distribution, etc.) have been evaluated. Some main results have emerged: for systems with a high content of minor actinides or minor plutonium isotopes, the major source of uncertainties is associated with $\mathrm{Pu} 238$, Pu241, and Am242M fission. Pu240 capture also plays an important role for almost all integral parameters. In general for sodium cooled fast reactors the uncertainty is dominated by the U238 inelastic. However, in the case of the coolant void reactivity, Na23 elastic and inelastic, U238 capture and the fission reaction in the $\mathrm{Pu}$ isotopes also make major contributions.

The difference with respect to the previous study of Subgroup 26 (where the BOLNA [3] covariance matrix was used) has been found in some cases be significant [4].

However, this paper will focus on target accuracy studies. In particular, besides providing the major finding in accuracies needed to satisfy required target design values, one major (theoretical) issue will be investigated. This issue is related to the fact that in previous studies correlation terms have been neglected in target accuracy assessments. As it will be seen later, this can have a very large impact on the final results.

We will first illustrate the theory behind the target accuracy assessment, then provide results for applications to the advanced fast reactor systems considered in the NEA/WPEC Subgroup 26 study, and finally provide some indications on how to proceed for future studies.

\section{THEORY}

Using a covariance data matrix (i. e. standard deviations on diagonal, and correlations on off-diagonal term) $\boldsymbol{D}$ and sensitivity coefficient arrays $\boldsymbol{S}_{\boldsymbol{R}}$ for an integral parameter $\boldsymbol{R}$, one can calculate the uncertainty $\Delta \boldsymbol{R}^{2}$ on the integral parameter using the sandwich formula [4]:

$$
\Delta R^{2}=S_{R}^{+} D S_{R}
$$

A successive step is the assessment of target accuracy requirements Target accuracy assessments [5] are the inverse problem of the uncertainty evaluation. To establish priorities and target accuracies on data uncertainty reduction, a formal approach can be adopted by defining target accuracy on design parameter and finding out required accuracy on data. In fact, the unknown uncertainty data requirements can be obtained by solving a 
International Conference on Nuclear Data for Science and Technology 2010

minimization problem where the sensitivity coefficients in conjunction with the existing constraints provide the needed quantities to find the solutions.

The unknown uncertainty data requirements $\boldsymbol{d}_{\boldsymbol{i}}$ can be obtained (e.g., for variables $i$ not correlated among themselves), by solving the following minimization problem for the functional $\boldsymbol{Q}$ :

$$
\boldsymbol{Q}=\sum_{i} \lambda_{i} / \boldsymbol{d}_{\boldsymbol{i}}^{2}=\min \quad \boldsymbol{i}=1 \ldots \boldsymbol{I}
$$

with the following constraints:

$$
\sum_{i} S_{n i}^{2} d_{i}^{2} \leq\left(R_{n}^{T}\right)^{2} \quad n=1 \ldots N
$$

where $N$ is the total number of integral design parameters, $S_{n i}$ are the sensitivity coefficients for the integral parameter $\boldsymbol{R}_{\boldsymbol{n}}$ and $\boldsymbol{R}_{n}^{\boldsymbol{T}}$ are the target accuracies on the $\boldsymbol{N}$ integral parameters; $\lambda_{i}$ are "cost" parameters related to each $\sigma_{i}$ and should give a relative figure of merit of the difficulty of improving that parameter (e.g., reducing uncertainties with an appropriate experiment).

In practical cases, in order to limit the number of variables (we remind that the unknown $\boldsymbol{d}_{\boldsymbol{i}}$ variables are the standard deviations of the cross-sections for which target accuracies are required) and make the problem feasible, the number $\boldsymbol{I}$ is obtained by selecting the variables based on their contribution to the uncertainties; for instance, by selecting only those which globally account at least for a fixed quantity (e. g. $98 \%$ as done in [2]) of the overall uncertainty for each integral parameter.

All target accuracy assessments performed up to now did not account for correlations between the data. This assumption is, of course, rather arbitrary, but it is acceptable for scoping studies in early phases of reactor design.

In the present paper we decided to investigate what kind of impact this assumption can make on final target accuracy requirements.

When taking into account correlations the constraints of Eq. (3) become:

$$
\sum_{i} G_{i}^{n}+\sum_{i i^{\prime}} C_{i i^{\prime}}^{n}+\sum_{i} F_{i}^{n}+P_{n} \leq\left(R_{n}^{T}\right)^{2} \quad n=1 \ldots N
$$

(4)

where $\boldsymbol{G}_{\boldsymbol{i}}^{\boldsymbol{n}}$, as in Eq. (3), represents the uncertainty related to the standard deviations of the selected variables:

$$
G_{i}^{n}=S_{n i}^{2} d_{i}^{2}
$$

$\boldsymbol{C}_{\boldsymbol{i}}^{\boldsymbol{n}}$, are the correlation terms among the selected variables with both $\boldsymbol{i}$ and $\boldsymbol{i}$ ' going from 1 to...I:

$$
C_{i i^{\prime}}^{n}=S_{n i} d_{i} \operatorname{Corr}_{i i^{\prime}} d_{i^{\prime}} S_{n i^{\prime}}^{+}
$$

$\boldsymbol{C o r r}_{i \boldsymbol{i}}$, is the correlation value between variable $\boldsymbol{i}$ and $\boldsymbol{i}$. $\boldsymbol{F}_{\boldsymbol{i}}^{\boldsymbol{n}}$ are the correlations among the selected and unselected variables:

$$
F_{i}^{n}=S_{n i} d_{i} \operatorname{Corr}_{i j} d_{j} S_{n j} \quad j=1 \ldots K
$$

where $\boldsymbol{d}_{j}$ are standard deviations that are not variables but considered constants and $\boldsymbol{K}$ is the total number of constant terms that are correlated to variable $\boldsymbol{i}$. Finally, $\boldsymbol{P}_{\boldsymbol{n}}$ represents the constant residual uncertainty for integral parameter $\boldsymbol{R}_{\boldsymbol{n}}$ due to the unselected variables. As can be seen, in the end we consider only the standard deviations of the selected cross sections as variables keeping the correlation values constant.

In order to solve the nonlinear minimization problem with nonlinear constraints we have used the SNOPT code [6]. SNOPT uses a sequential quadratic programming (SQP) algorithm that obtains search directions from a sequence of quadratic programming subproblems. Each QP subproblem minimizes a quadratic model of a certain Lagrangian function subject to a linearization of the constraints. An augmented Lagrangian merit function is reduced along each search direction to ensure convergence from any starting point.

As many other optimization codes SNOPT needs the problem Jacobian. The code can calculate derivatives in a numerical manner, but our experience is that it better performs both in execution time and accuracy when derivatives are directly provided by the user. Derivatives with respect to variable $\boldsymbol{d}_{\boldsymbol{i}}$ of Equation (2) and (4) can be analytically calculated so their corresponding analytical formulation was implemented in the user function subroutines that were provided to SNOPT.

\section{TARGET ACCURACY ASSESSMENT FOR ADVANCED FAST REACTOR SYSTEM}

As for the practical application we have chosen as reactor design those of fast reactor system that were used in [2], and in particular the EFR (Na-cooled European Fast Reactor), GCFR (Gas Cooled Fast Reactor), SFR (Sodium Fast Reactor), LFR (Lead Fast Reactor), ADMAB (Accelerator Driven Minor Actinide Burner). Details about these systems are reported in [2]. As far as covariance data we have used the new available AFCI 1.2 [1]. One major difference of this matrix with respect to the BOLNA one used in the the NEA/WPEC Subgroup 26 study, is the energy group structure adopted that consists in 33 energy groups (see table 1) mostly intended for fast reactor applications. As will be seen later, this has large implications on the final results. As for integral parameter we have used only the multiplication factor $\mathrm{K}_{\text {eff }}$ in order to simplify calculations and better understand the obtained results. For the target value on the integral parameter the value of $300 \mathrm{pcm}$ has been adopted (as in reference [2]) for all fast systems. 
International Conference on Nuclear Data for Science and Technology 2010

Table 1.33 energy group structure $(\mathrm{eV})$.

\begin{tabular}{|c|c|c|c|c|c|}
\hline Group & Up Ener. & Group & Up Ener. & Group & Up Ener. \\
\hline 1 & $1.9610^{7}$ & 12 & $6.7410^{4}$ & 23 & $3.0410^{2}$ \\
\hline 2 & $1.0010^{7}$ & 13 & $4.0910^{4}$ & 24 & $1.4910^{2}$ \\
\hline 3 & $6.0710^{6}$ & 14 & $2.4810^{4}$ & 25 & $9.1710^{1}$ \\
\hline 4 & $3.6810^{6}$ & 15 & $1.5010^{4}$ & 26 & $6.7910^{1}$ \\
\hline 5 & $2.2310^{6}$ & 16 & $9.1210^{3}$ & 27 & $4.0210^{1}$ \\
\hline 6 & $1.3510^{6}$ & 17 & $5.5310^{3}$ & 28 & $2.2610^{1}$ \\
\hline 7 & $8.2110^{5}$ & 18 & $3.3510^{3}$ & 29 & $1.3710^{1}$ \\
\hline 8 & $4.9810^{5}$ & 19 & $2.0310^{3}$ & 30 & $8.3210^{0}$ \\
\hline 9 & $3.0210^{5}$ & 20 & $1.2310^{3}$ & 31 & $4.0010^{0}$ \\
\hline 10 & $1.8310^{5}$ & 21 & $7.4910^{2}$ & 32 & $5.4010^{-1}$ \\
\hline 11 & $1.1110^{5}$ & 22 & $4.5410^{2}$ & 33 & $1.0010^{-1}$ \\
\hline
\end{tabular}

Table 2 shows the results of the target accuracy assessment for the EFR with and without taking into account correlations terms. For the meaning of the different components ( $\boldsymbol{G}, \boldsymbol{C}, \boldsymbol{F}$, and $\boldsymbol{P}$ ), we refer to Eq. (4). $\boldsymbol{V}$ is the partial total $(\boldsymbol{V}=\boldsymbol{G}+\boldsymbol{C}+\boldsymbol{F})$ and represents the total uncertainty related to the selected parameters. All the sums are performed in a statistical way (square root of sum of squares). The calculation without correlation has no residual term $\boldsymbol{P}$ as it was done in [2].

Table 2. Target accuracy assessment for EFR (pcm). 146 parameters selected.

\begin{tabular}{|c|c|c|c|c|c|c|c|}
\cline { 3 - 8 } \multicolumn{2}{c|}{} & $\boldsymbol{G}$ & $\boldsymbol{C}$ & $\boldsymbol{F}$ & $\boldsymbol{V}$ & $\boldsymbol{P}$ & Total \\
\hline \multirow{2}{*}{$\begin{array}{c}\text { No } \\
\text { corr. }\end{array}$} & Initial & $\mathbf{6 3 1}$ & - & - & $\mathbf{6 3 1}$ & - & $\mathbf{6 3 1}$ \\
\cline { 2 - 8 } & Final & $\mathbf{3 0 0}$ & - & - & $\mathbf{3 0 0}$ & - & $\mathbf{3 0 0}$ \\
\hline \multirow{2}{*}{$\begin{array}{c}\text { With } \\
\text { corr. }\end{array}$} & Initial. & $\mathbf{6 3 1}$ & $\mathbf{1 0 4 4}$ & $\mathbf{1 6 4}$ & $\mathbf{1 2 3 1}$ & $\mathbf{2 4 8}$ & $\mathbf{1 2 5 6}$ \\
\cline { 2 - 8 } & Final & $\mathbf{9 3}$ & $\mathbf{1 2 2}$ & $\mathbf{7 1}$ & $\mathbf{1 6 9}$ & $\mathbf{2 4 8}$ & $\mathbf{3 0 0}$ \\
\hline
\end{tabular}

One can immediately observe that the calculation with correlation requires a severe reduction on term $\boldsymbol{G}$ with more than a factor 3 . This is equivalent to a factor 10 when we consider that terms are combined using square values. This translates in drastic requirements on the single variables. Table 3 shows the corresponding requirements for the 5 most important, in terms of contribution to total uncertainty, variables for the case with and without uncertainties.

Table 3. EFR target accuracy requirements on 5 most important variables. Standard deviations (\%).

\begin{tabular}{|c|c|c|c|}
\hline Variable & Initial & No Correl. & With Correl. \\
\hline U8 $\sigma^{\text {inel }}$ Gr. 5 & $\mathbf{2 0 . 6}$ & $\mathbf{3 . 8}$ & $\mathbf{0 . 5}$ \\
\hline U8 $\sigma^{\text {inel }}$ Gr. 4 & $\mathbf{1 9 . 4}$ & $\mathbf{3 . 2}$ & $\mathbf{0 . 5}$ \\
\hline U8 $\sigma^{\text {inel }}$ Gr. 6 & $\mathbf{1 6 . 9}$ & $\mathbf{4 . 4}$ & $\mathbf{0 . 6}$ \\
\hline U8 $\sigma^{\text {inel }}$ Gr. 3 & $\mathbf{2 0 . 1}$ & $\mathbf{5 . 5}$ & $\mathbf{0 . 7}$ \\
\hline Pu1 $\sigma^{\text {fis }}$ Gr. 10 & $\mathbf{2 0 . 0}$ & $\mathbf{6 . 4}$ & $\mathbf{0 . 9}$ \\
\hline
\end{tabular}

The requirements imposed by taking into account the correlations are very small (sometimes an order of magnitude less than the corresponding case with no correlation) and probably beyond achievable limits with current experimental techniques. This effect is also enhanced by the fact that the energy group structure adopted is too detailed so that, due to energy correlations to be kept constant, it will impose very stringent requirement for variables in a specific range of energy. One lesson learned is, therefore, that a broader energy structure should be adopted for this type of study. Moreover, as these results translate in recommendations to evaluators and cross section measurement experts, it appears also wise that this kind of information is provided on a broader basis of energy dependence.

However, since correlations have a profound impact on target accuracy requirements, in future studies correlations have to be taken into account when performing target accuracy assessments.

In table 4 through 11 we show the results obtained for the corresponding cases of the other fast systems. As for the previous case of the EFR, the results confirm the importance of the contribution coming from the correlation terms and their significant impact on the final results of the target accuracy assessment

Table 4. Target accuracy assessment for GFCR (pcm). 173 parameters selected.

\begin{tabular}{|c|c|c|c|c|c|c|c|}
\cline { 3 - 8 } \multicolumn{2}{c|}{} & $\boldsymbol{G}$ & $\boldsymbol{C}$ & $\boldsymbol{F}$ & $\boldsymbol{V}$ & $\boldsymbol{P}$ & Total \\
\hline \multirow{2}{*}{$\begin{array}{c}\text { No } \\
\text { corr. }\end{array}$} & Initial & $\mathbf{9 8 2}$ & - & - & $\mathbf{9 8 2}$ & - & $\mathbf{9 8 2}$ \\
\cline { 2 - 8 } & Final & $\mathbf{3 0 0}$ & - & - & $\mathbf{3 0 0}$ & - & $\mathbf{3 0 0}$ \\
\hline \multirow{2}{*}{$\begin{array}{l}\text { With } \\
\text { corr. }\end{array}$} & Initial. & $\mathbf{9 8 2}$ & $\mathbf{1 6 4 0}$ & $\mathbf{1 7 4}$ & $\mathbf{1 9 1 9}$ & $\mathbf{2 7 8}$ & $\mathbf{1 9 3 9}$ \\
\cline { 2 - 8 } & Final & $\mathbf{5 2}$ & $\mathbf{8 1}$ & $\mathbf{5 9}$ & $\mathbf{1 1 3}$ & $\mathbf{2 7 8}$ & $\mathbf{3 0 0}$ \\
\hline
\end{tabular}

Table 5. GFCR target accuracy requirements on 5 most important variables. Standard deviations (\%).

\begin{tabular}{|c|c|c|c|}
\hline Variable & Initial & No Correl. & With Correl. \\
\hline U8 $\sigma^{\text {inel }}$ Gr. 5 & $\mathbf{2 0 . 6}$ & $\mathbf{2 . 1}$ & $\mathbf{0 . 3}$ \\
\hline U8 $\sigma^{\text {inel }}$ Gr. 4 & $\mathbf{1 9 . 4}$ & $\mathbf{2 . 3}$ & $\mathbf{0 . 3}$ \\
\hline U8 $\sigma^{\text {inel }}$ Gr. 3 & $\mathbf{2 0 . 1}$ & $\mathbf{3 . 1}$ & $\mathbf{0 . 4}$ \\
\hline U8 $\sigma^{\text {inel }}$ Gr. 6 & $\mathbf{1 6 . 9}$ & $\mathbf{3 . 1}$ & $\mathbf{0 . 4}$ \\
\hline Pu1 $\sigma^{\text {fis }}$ Gr. 10 & $\mathbf{2 0 . 0}$ & $\mathbf{4 . 2}$ & $\mathbf{0 . 5}$ \\
\hline
\end{tabular}

Table 6. Target accuracy assessment for SFR (pcm). 201 parameters selected.

\begin{tabular}{|c|c|c|c|c|c|c|c|}
\cline { 3 - 8 } \multicolumn{2}{c|}{} & $\boldsymbol{G}$ & $\boldsymbol{C}$ & $\boldsymbol{F}$ & $\boldsymbol{V}$ & $\boldsymbol{P}$ & Total \\
\hline \multirow{2}{*}{$\begin{array}{c}\text { No } \\
\text { corr. }\end{array}$} & Initial & $\mathbf{9 0 3}$ & - & - & $\mathbf{9 0 3}$ & - & $\mathbf{9 0 3}$ \\
\cline { 2 - 8 } & Final & $\mathbf{3 0 0}$ & - & - & $\mathbf{3 0 0}$ & - & $\mathbf{3 0 0}$ \\
\hline \multirow{2}{*}{$\begin{array}{l}\text { With } \\
\text { corr. }\end{array}$} & Initial. & $\mathbf{9 0 3}$ & $\mathbf{1 8 5 8}$ & $\mathbf{1 8 6}$ & $\mathbf{2 0 7 4}$ & $\mathbf{2 5 1}$ & $\mathbf{2 0 9 0}$ \\
\cline { 2 - 8 } & Final & $\mathbf{8 0}$ & $\mathbf{1 2 1}$ & $\mathbf{7 6}$ & $\mathbf{1 6 4}$ & $\mathbf{2 5 1}$ & $\mathbf{3 0 0}$ \\
\hline
\end{tabular}

Table 7. SFR target accuracy requirements on 5 most important variables. Standard deviations (\%).

\begin{tabular}{|c|c|c|c|}
\hline Variable & Initial & No Correl. & With Correl. \\
\hline Pu $8 \sigma^{\text {fis }}$ Gr. 7 & $\mathbf{5 0 . 0}$ & $\mathbf{4 . 7}$ & $\mathbf{0 . 9}$ \\
\hline Pu $8 \sigma^{\text {fis }}$ Gr. 8 & $\mathbf{5 0 . 0}$ & $\mathbf{4 . 8}$ & $\mathbf{0 . 9}$ \\
\hline Pu $8 \sigma^{\text {fis }}$ Gr. 6 & $\mathbf{5 0 . 0}$ & $\mathbf{5 . 1}$ & $\mathbf{1 . 0}$ \\
\hline Pu $1 \sigma^{\text {fis }}$ Gr. 10 & $\mathbf{2 0 . 0}$ & $\mathbf{3 . 6}$ & $\mathbf{0 . 7}$ \\
\hline Pu $8 \sigma^{\text {fis }}$ Gr. 9 & $\mathbf{5 0 . 0}$ & $\mathbf{6 . 2}$ & $\mathbf{1 . 1}$ \\
\hline
\end{tabular}


International Conference on Nuclear Data for Science and Technology 2010

Table 8. Target accuracy assessment for LFR (pcm). 163 parameters selected.

\begin{tabular}{|c|c|c|c|c|c|c|c|}
\cline { 2 - 8 } \multicolumn{2}{c|}{} & $\boldsymbol{G}$ & $\boldsymbol{C}$ & $\boldsymbol{F}$ & $\boldsymbol{V}$ & $\boldsymbol{P}$ & Total \\
\hline \multirow{2}{*}{$\begin{array}{c}\text { No } \\
\text { corr. }\end{array}$} & Initial & $\mathbf{8 0 9}$ & - & - & $\mathbf{8 0 9}$ & - & $\mathbf{8 0 9}$ \\
\hline \multirow{2}{*}{$\begin{array}{l}\text { With } \\
\text { corr. }\end{array}$} & Initial. & $\mathbf{8 0 9}$ & $\mathbf{1 4 1 6}$ & $\mathbf{1 5 0}$ & $\mathbf{1 6 3 8}$ & $\mathbf{2 0 5}$ & $\mathbf{1 6 5 1}$ \\
\cline { 2 - 8 } & Final & $\mathbf{1 2 0}$ & $\mathbf{1 7 0}$ & $\mathbf{6 8}$ & $\mathbf{2 1 9}$ & $\mathbf{2 0 5}$ & $\mathbf{3 0 0}$ \\
\hline
\end{tabular}

Table 9. LFR target accuracy requirements on 5 most important variables. Standard deviations (\%).

\begin{tabular}{|c|c|c|c|}
\hline Variable & Initial & No Correl. & With Correl. \\
\hline U8 $\sigma^{\text {inel }}$ Gr. 5 & $\mathbf{2 0 . 6}$ & $\mathbf{3 . 0}$ & $\mathbf{0 . 8}$ \\
\hline Pu8 $\sigma^{\text {fis }}$ Gr. 7 & $\mathbf{5 0 . 0}$ & $\mathbf{5 . 5}$ & $\mathbf{1 . 4}$ \\
\hline U8 $\sigma^{\text {inel }}$ Gr. 6 & $\mathbf{1 6 . 9}$ & $\mathbf{3 . 2}$ & $\mathbf{0 . 8}$ \\
\hline Pu8 $\sigma^{\text {fis }}$ Gr. 8 & $\mathbf{5 0 . 0}$ & $\mathbf{5 . 9}$ & $\mathbf{1 . 4}$ \\
\hline U8 $\sigma^{\text {inel }}$ Gr. 4 & $\mathbf{1 9 . 4}$ & $\mathbf{3 . 9}$ & $\mathbf{1 . 0}$ \\
\hline
\end{tabular}

Table 10. Target accuracy assessment for ADMAB (pcm). 270 parameters selected.

\begin{tabular}{|c|c|c|c|c|c|c|c|}
\cline { 3 - 8 } \multicolumn{2}{c|}{} & $\boldsymbol{G}$ & $\boldsymbol{C}$ & $\boldsymbol{F}$ & $\boldsymbol{V}$ & $\boldsymbol{P}$ & Total \\
\hline \multirow{2}{*}{$\begin{array}{c}\text { No } \\
\text { corr. }\end{array}$} & Initial & $\mathbf{1 1 2 4}$ & - & - & $\mathbf{1 1 2 4}$ & - & $\mathbf{1 1 2 4}$ \\
\hline \multirow{2}{*}{$\begin{array}{l}\text { With } \\
\text { corr. }\end{array}$} & Initial. & $\mathbf{1 1 2 4}$ & $\mathbf{2 1 2 5}$ & $\mathbf{1 9 4}$ & $\mathbf{2 4 1 1}$ & $\mathbf{2 5 7}$ & $\mathbf{2 4 2 5}$ \\
\cline { 2 - 8 } & Final & $\mathbf{7 2}$ & $\mathbf{1 1 9}$ & $\mathbf{6 8}$ & $\mathbf{1 5 5}$ & $\mathbf{2 5 7}$ & $\mathbf{3 0 0}$ \\
\hline
\end{tabular}

Table 11. ADMAB target accuracy requirements on 5 most important variables. Standard deviations (\%).

\begin{tabular}{|c|c|c|c|}
\hline Variable & Initial & No Correl. & With Correl. \\
\hline Am $1 \sigma^{\text {fis }}$ Gr. 6 & $\mathbf{1 0 . 0}$ & $\mathbf{1 . 6}$ & $\mathbf{0 . 4}$ \\
\hline Am $1 \sigma^{\text {fis }}$ Gr. 5 & $\mathbf{1 0 . 0}$ & $\mathbf{1 . 7}$ & $\mathbf{0 . 4}$ \\
\hline Pu1 $\sigma^{\text {fis }}$ Gr. 10 & $\mathbf{2 0 . 0}$ & $\mathbf{2 . 8}$ & $\mathbf{0 . 5}$ \\
\hline Pu1 $\sigma^{\text {fis }}$ Gr. 9 & $\mathbf{1 8 . 9}$ & $\mathbf{2 . 8}$ & $\mathbf{0 . 5}$ \\
\hline Cm $5 \sigma^{\text {fis }}$ Gr. 10 & $\mathbf{5 0 . 0}$ & $\mathbf{4 . 8}$ & $\mathbf{0 . 9}$ \\
\hline
\end{tabular}

\section{CONCLUSIONS}

We have performed a target accuracy assessment using new available covariance data, the AFCI 1.2 covariance data matrix. At the same time, the more theoretical issue of taking into account correlation terms has been deeply investigated. The major conclusions and recommendations obtained by this study are:

- The impact of correlation terms is very significant in target accuracy assessment evaluation and produces very stringent requirements on nuclear data

- For this type of study a broader energy group structure should be used, in order to smooth out requirements and provide better information to evaluators and cross section measurement experts

- The main difference in results between using
BOLNA or AFCI 1.2 covariance data are related to minor actinides, minor $\mathrm{Pu}$ isotopes, structural materials (in particular Fe56), and coolant isotopes (Na23)

For future target accuracy requirements study a possible broader energy structure could be the one we propose in table 12 that contains 6 energy groups. This structure covers the entire spectrum and could be used for studies of fast, epithermal, and thermal reactors.

Table 12. Proposed 6 energy groups structure $(\mathrm{eV})$.

\begin{tabular}{|c|c|c|c|}
\hline Group & Up Energy & Group & Up Energy \\
\hline $\mathbf{1}$ & $\mathbf{1 . 9 6 4 0 3 ~ 1 0}^{\mathbf{7}}$ & $\mathbf{4}$ & $\mathbf{2 . 0 3 4 6 8 ~ 1 0}^{\mathbf{3}}$ \\
\hline $\mathbf{2}$ & $\mathbf{4 . 9 7 8 7 1 ~ 1 0}^{\mathbf{5}}$ & $\mathbf{5}$ & $\mathbf{2 . 2 6 0 3 3 ~ 1 0}^{\mathbf{1}}$ \\
\hline $\mathbf{3}$ & $\mathbf{6 . 7 3 7 9 5 1 0 ^ { 4 }}$ & $\mathbf{6}$ & $\mathbf{5 . 4 0 0 0 0} \mathbf{1 0}^{-1}$ \\
\hline
\end{tabular}

The energy limits are based on physical consideration with bands that cover the region above the threshold of fertile isotope fission cross-sections, and of many inelastic cross-sections, the region of the continuum down to the upper unresolved resonance energy limit, the unresolved resonance energy region, the resolved resonance region, the epithermal range, and thermal range.

\section{ACKNOWLEDGMENTS}

Prepared for the U.S. Department of Energy through the INL LDRD Program under DOE Idaho Operations Office Contract DE-AC07-05ID14517.

\section{REFERENCES}

[1] P.Oblozinsky, C.M. Mattoon, M. Herman, S.F. Mughabghab, M.T. Pigni National Nuclear Data Center, (BNL), P. Talou, G.M. Hale, A.C. Kahler, T. Kawano, R.C. Little, P.G. Young, LANL, Los Alamos, "Progress on Nuclear Data Covariances: AFCI-1.2 Covariance Library", Tech. Rep, BNL-90897-2009, Brookhaven National Laboratory, 2009

[2] "OECD/NEA WPEC Subgroup 26 Final Report: Uncertainty and Target Accuracy Assessment for Innovative Systems Using Recent Covariance Data Evaluations", 2008.

[3] D. Rochman, M. Herman, P. Oblozinsky and. S. F. Mughabghab, "Preliminary Cross-Section Covariances for WPEC Subgroup 26," Tech. Rep. BNL-77407-2007-IR, Brookhaven National Laboratory, 2007.

[4] G. Palmiotti, M. Salvatores, M. Assawaroongruengchot, "Nuclear Data for Innovative Fast Reactors: Impact of Uncertainties and New Requirements", International Conference on Fast Reactors and Related Fuel Cycles (FR09), December 7 - 11, 2009, Kyoto, Japan

[5] G. Palmiotti, M. Salvatores, "Use of Integral Experiments in the Assessment of Large Liquid-Metal Fast Breeder Reactor Basic Design Parameters," Nucl. Sci. Eng. 87, 333, 1984

[6] Philip E. Gill, Walter Murray, and Michael A. Saunders. SNOPT: An SQP algorithm for Large-Scale constrained programming. Technical Report SOL 97-3, Systems Optimization Laboratory, Department of Operations Research, Stanford University, Stanford, California 94305-4022, 1997. 